\title{
Intergrated SMA Soft Actuators with Periodic and Inhomogeneous Deformations by Modulating Elastic Tendon Structures
}

Dachao Li ${ }^{1 \#}$, Dong Niü ${ }^{1 \#}$, Guoyong Ye ${ }^{1}$, Biao Lei ${ }^{1}$, Weitao Jiang ${ }^{1}$, Feng Luo ${ }^{3}$, Jinju Chen ${ }^{4}$, Xin

$$
L i^{2} \text {, Shaoxing } Q u^{5} \text {, Hongzhong Liu }{ }^{1,6^{*}}
$$

D. Li, Dr. D. Niu, Prof. G.Ye, Dr. B. Lei, Prof. W. Jiang, Prof. F. Luo, Prof. J. Chen, Prof. X. Li, Prof. S. Qu, Prof. H. Liu.

${ }^{1}$ State Key Laboratory for Manufacturing Systems Engineering, Xi'an Jiaotong University, Xi'an 710049, China

${ }^{2}$ Department of Microelectronics, School of Electronics and Information Engineering, Xi'an Jiaotong University, Xi'an 710049, China

${ }^{3}$ IMDEA Nanoscience, Faraday 9, Ciudad Universitaria de Cantoblanco, 28049, Madrid, Spain

${ }^{4}$ School of Engineering, Stephenson Building Newcastle University, UK

${ }^{5}$ Department of Engineering Mechanics, Zhejiang University, Hangzhou 310027, China

${ }^{6}$ Shaanxi Joint Key Laboratory of Graphene, Xi'an 710049, China

\section{Abstract}

The soft actuators similar to the periodic motion of biological organisms have been accomplished based on assembling multiple unidirectional memory effect SMA components or introducing biasing elements, which give rise to the complex structure and difficult control of soft actuators. Here, inspired by the Power amplified biological systems, beneficial with the advantages of 3D integrated molding technology, we proposed an integrated SMA-PDMS composite structure (SPCS), which can achieve periodic heterogeneous actuation only by controlling the SMA phase transformation and the PDMS strain potential energy distribution states. To test the feasibility of

\footnotetext{
\# These authors contribute equally to this work.

*Corresponding author: hzliu@mail.xjtu.edu.cn.
} 
the mechanism, we conducted a theoretical model of SPCS deformation. The results of numerical feasibility analysis showed that the factors affecting SPCS deformation mainly involve the excitation current strength of SMA 、 PDMS structure thickness and its distribution state. The experimental results show that the current intensity mainly affects the deformation rate of SPCS, and the thickness of PDMS is not only the key to realize the periodic deformation of SPCS, but also the orderly arrangement of PDMS structure thickness is helpful for SPCS to achieve periodic heterogeneous deformation. These demonstrated that the proposed mechanism can inspire the design of soft actuators, smart wearable equipment, and medical devices.

\section{Introduction}

Soft robots, which are composed of soft materials and inspired by animal brilliant functionalities, have the potentials in achieving multimodal and adaptive deformation with good mechanical compliance, manipulating and grasping fragile objects with gentle human-machine interactions, and so on. ${ }^{[1-5]}$ Unlike the traditional rigid robots assembled with rigid links and flexible joints, the actuation of soft robots is fundamentally based on the stimulus-responsive soft materials, ${ }^{[6-10]}$ EAP (electroactive polymers), ${ }^{[11-15]}$ fluidic/pneumatic elastic elements, ${ }^{[16-19]}$ heat/chemical triggered hydrogels, ${ }^{[20,21]}$ magnetic memory composites, ${ }^{[22-24]}$ SMA (shape memory alloys). ${ }^{[25,26]}$ Given the advantages in high power to weight ratios and low power consumption, SMA (shape memory alloys) based soft actuators enable combining the heat triggered shape memory effect of SMA and the good flexibilities of soft matrix. ${ }^{[27,28]}$ Some significant efforts have been reported in soft robots, exoskeleton assisted rehabilitation devices, shape morphing wings, etc. Although impressive, these implementations are commonly based on commercial SMA elements with a one-way memory effect, only giving rise to deformation in one direction and cannot complete periodic deformation. 
However, in nature, many complex motions such as the swimming of a fish and crawling of a worm are caused by the periodic deformation of muscular actuators. So, realizing periodic actuation with the commercial SMA is of urgency in developing SMA based soft actuators.

Currently, most of the SMA-based actuators achieve Periodic actuation with the help of biasing elements, such as springs, elastic plates. The SMA-actuator based on the biasing element mainly realizes the Periodic actuation by releasing the strain potential energy of the bias components, such as spring, thin polyvinyl chloride (PVC) polymer plate, glass fiber cloth, and elastic polymer sheet, etc., ${ }^{[29-33]}$ and realized continuous bending deformation similar to waves through linear combination. However, it cannot be ignored that introducing biasing elements may result in structural complexities in SMA soft actuator design. Due to the high modulus characteristics of the biasing element, the actuator may present a significant challenge for achieving a large deformation.

In addition, the actuator can achieve Periodic deformation by assembling in multiple SMA components that are different from the actuator based on biasing elements. It mainly realizes the Periodic actuation by alternately activating SMA wires arranged in an antagonist-protagonist configuration. E.g. the actuator Periodic actuation realized by Joule heat alternately actuating SMA contraction. ${ }^{[34,35]}$ Additionally, it can also be accomplished by alternating excitation of coupled SMA with infrared light. ${ }^{[9]}$ The key to Periodic actuation is the arrangement of the coupled SMA wires, which will bring challenges to simplifying the structural arrangement and precise control of the actuator. In addition, distortion and wrinkle deformation are inevitable during the actuating process, which may also put forward better requirements for the control of mechanical deformation accuracy. Therefore, based on simplifying the SMA layout, how to improve the controllability and sequence of the software has attracted more and more attention. E.g. Saeed Akbar, et al. exploited 
the great manufacturing flexibility of multi-material 3D printing to fabricate various bending soft actuators by precisely controlling the spatial arrangements of different printing materials with different stiffnesses. ${ }^{[36]}$ However, it cannot be ignored that these still has certain challenges in achieving periodic deformation.

Here, based on simplifying the SMA layout, it is the focus of this paper to improve the controllability and sequence performance of soft actuators by optimizing the distribution of soft matrix structure thickness, while achieving the periodic heterogeneous deformation. With the help of 3D integrated molding technology, the soft actuator was designed and manufactured as a SMAPDMS composite structure (SPCS), which requires neither complicated SMA layout designs or an additional biasing component to achieve periodic heterogeneous actuation. First, the SPCS relies on SMA to actuate bending, but the power to restore the deformation is derived from the strain potential energy stored inside the structure itself. In addition, beneficial with the integrated design and manufacturing of 3D printing technology, the soft actuators achieved diverse and Periodic deformation by optimizing the strain potential energy distribution state of the PDMS matrix by improving the PDMS structure thickness based on the simplified SMA layout, thereby improved the controllable and deformation performance of the soft actuator. According to the numerical feasibility analysis and experimental data, it can be concluded that increasing the current has little effect on the maximum deformation of the SPCS but will improve the mechanical deformation efficiency of the SPCS during the actuation process. In addition, although the increase in the structural thickness of PDMS limited the deformation of the SPCS, it increased the recovery performance. Besides, it can be seen from the experimental data of the PDMS structural optimization that both depth and shape of the PDMS structure promoted the local deformation ("joint") of the SPCS, but also the structure position also affected its deformation order. 


\section{Materials and Methods}

\subsection{Materials}

A commercial implementation of nickel-titanium alloy wires with a diameter of $0.5 \mathrm{~mm}(\mathrm{Ni}: 55$ $\mathrm{wt} \%$, Ti: $45 \mathrm{wt} \%$ ) used throughout this research. The SMA wires were selected based on the phase transformation temperature $\left(45^{\circ} \mathrm{C}\right)$ being above the room temperature in the useful temperature range of the chosen polymer, but easily attainable through joule heating. Compared with other types of SMA wire, it has a good strain recovery capacity, stable transformation temperatures with a relatively high electrical resistance. The detailed parameters of SMA materials provided by the manufacturer are shown in Table 1 depicted in Supporting Information. PDMS is prepared as an energy storage element of the SPCS. The PDMS obtained from Dow Corning (Sylgard 184) is used as the host matrix. PDMS is a two-part solvent-free flexible silicone organic polymer (Mixed with a base-to-catalyst ratio of 10:1) in the form of a base compound with a separate hydrosilane curing agent that acts as a crosslinker. The physical properties of PDMS are listed in Table 2 depicted in Supporting Information.

\subsection{Characterizations}

The SPTS were actuated using a custom-made current control board connected to a power source, which allows for the control of current input patterns to induce joule heating effect into SMA wires. The current intensity was calibrated using a multimeter. FLIR SC7300M infrared camera was employed to record the real-time temperature of the SPCS surface under different current to reveal the heat transfer, electrothermal conversion of the SMA wires inner the PDMS matrix. The actuation process of SPTS was recorded by a High Definition recorder to explore the influence of current and structure PDMS on the deformation of SPCS. Finite element software COMSOL Multiphysics 5.4 carried out numerical analysis on the deformation process of SPCS. 


\section{Results and discussion}

\subsection{Fabrication of SPCS}

To fabricate the SPCS, one SMA wires, the local structures, PDMS, and casting mold are required (Figure 1). First, take advantage of the customizability of integrating 3D printing technology, a pair of resin mold with the desired local structure was designed and prepared (Figure 1(a)). The PDMS, as strain potential energy storage materials, is mixed with a base-to-catalyst ratio of 10:1, degassed to remove all bubbles, and accurately injected into the structure of the mold with automatic dispensing (Figure 1(b)). Then, the PDMS with structures was completely cured by thermal curing on a $60^{\circ} \mathrm{C}$ hot plate for $10 \mathrm{~h}$ (Figure 1(c)). Next, the PDMS with heterogeneous was completed (Figure 1(d)). Finally, the SMA with a single memory effect was embedded in the elastic element, and the two ends of the SMA were fixed without sliding by using buckles (Figure 1(e)). The concrete structure of the SPCS, the location and arrangement of the SMA in the elastic element was shown in Figure $\mathbf{S 1}$ in the supporting information. The bionic gripper mentioned in Section 3.4 was also assembled from structured SPCS (Figure 1 (f)).

\subsection{Theoretical Model}

For our proposed SPCS, periodic and heterogeneous deformation of the soft actuator were mainly realized without complicated SMA layout designs or additional biasing components. To investigate the deformation behavior of the proposed SPCS actuator, a mathematical model was described based on the thermomechanical model of the SMA exhibiting large bending deformation. The mathematical model can be resolved by a theory similar to the classic flexible beam-shape theory ${ }^{[37-39]}$ and constructed as Equation 1.

$$
\frac{2\left(\theta-\theta_{i n i-}\right)}{\left(L_{\mathrm{SPCS}}-\Delta L_{\mathrm{SPCS}}\right)}=\frac{128 \sigma S \beta}{\mu \pi E_{\mathrm{PDMS}}\left(d^{4}-d_{i}^{4}\right)}
$$


Where $d_{i}$ and $d$ are the inner diameter and outer diameter of the PDMS respectively. $E_{P D M S}$ is Young's modulus of PDMS. $\sigma$ is the stress, $S$ is the cross-sectional area of the SMA wire. $\beta$ is the distance between the SMA wire and the neutral axis of SPCS. $L_{\mathrm{SPCS}}$ and $\Delta L_{\mathrm{SPCS}}$ are the length and increment of SMA wire respectively, and $\theta$ is the deformation angle of SPCS. According to the thermomechanical model, the main factors affecting the deformation of SPCS are the modulus and thickness of PDMS, and the stress and strain of the alloy. Therefore, we need to describe the constitutive relationship of the materials, such as SMA and PDMS.

SMA is a kind of material which undergoes phase transformation triggered by temperature and can change its shape, which provides the main driving force for SPCS deformation.Upon the Martensite Phase Fraction model (i.e., Equation 2), it was the temperature changes of SMA that induce the variations of Martensite Phase Fraction, which affected the stress-strain state of SMA wire during the actuation process. ${ }^{[40-42]}$ In this case, the relationship between the heat transfer process and heat triggered mechanical deformation could be successfully established, which is denoted as a thermo-constitutive model of SMA, as shown in Equation 3.

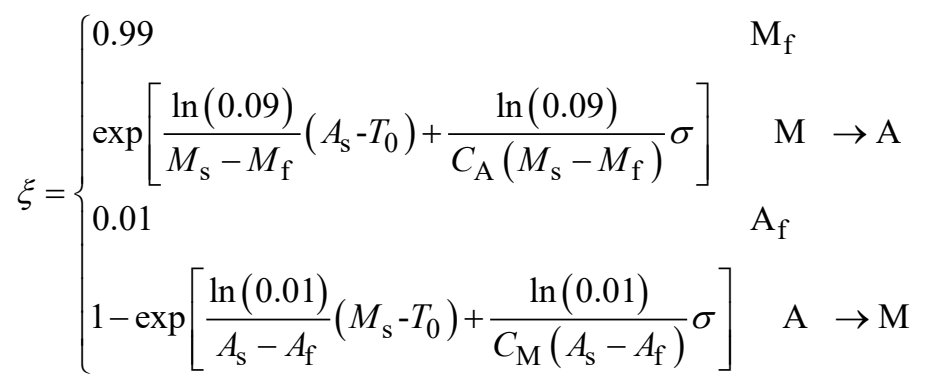

Where $A_{\mathrm{s}}$ and $A_{\mathrm{f}}$ are the Austenite Start and Austenite Finish temperature, respectively. $M_{\mathrm{s}}$ is the Martensite Start temperature, and $M_{\mathrm{f}}$ is Martensite Finish temperature. $C_{A}$ and $C_{M}$ are the stress influence coefluence in Austenite state and Martensite state, respectively. 


$$
\sigma=\varepsilon\left(E_{A}-\xi E_{A}+\xi E_{M}\right)-\varepsilon_{L}\left(\xi E_{A}-\xi^{2} E_{A}+\xi E_{M}\right)
$$

This form identifies the stress $(\sigma)$ on the SMA as a function of the strain $(\varepsilon)$ and the phase fraction $(\xi) . \varepsilon_{L}$ is the maximum residual strain. $E_{A}$ and $E_{M}$ are Young's modulus of SMA in the austenite phase and the martensite phase, respectively. Combining Equation (2) and (3), we can deduce a conclusion that the main factor affecting the stress-strain change of SMA is the SMA internal temperature.It could be attributed to the effect that the mutual transformation between Austenite $(A)$ crystal structure and Martensite $(M)$ crystal structure primarily highly depends on the SMA phase transformation temperature. As the SMA temperature exceeds austenite phase transformation temperature $\left(A_{\mathrm{s}}\right)$ under the Joule heat effect, the strain of SMA appears significant changes $^{[43]}$, which gives rise to the deformation of SPCS ( $A_{\mathrm{s}} \rightarrow A_{\mathrm{f}}$ in Figure $\mathbf{S 2}$ in supporting information).

The PDMS, as a kind of soft material, enables to release the strain potential energy, which played a key role in actuating the SPCS recovery deformation. Here, the Ogden model ${ }^{[44]}$ was chosen because it fits the experiment well and is accurate for numerical calculation for used materials. According to the Ogden model, the strain energy function $\psi$ is written in this form

$$
\psi=\sum_{i=1}^{N} \frac{\tau_{i}}{v_{i}}\left(\lambda_{1}^{v_{i}}+\lambda_{2}^{v_{i}}+\lambda_{3}^{v_{i}}-3\right)
$$

Where $\tau_{i}$ and $v_{i}$ are constants determined by the experiments, $\lambda_{N}$ are principle stretches. And the Cauchy stresses $\sigma_{\mathrm{c}}$ are derived in this form

$$
\sigma_{c n}=J^{-1} \lambda_{n} \frac{\partial \psi}{\partial \lambda_{n}}, \quad n=1,2,3
$$


The constants $\tau_{i}$ and $v_{i}$ of the PDMS are obtained by importing the measured stress-strain curve of PDMS into the Ogden model. Then the force of PDMS on SMA can be expressed as

$$
F=\sigma_{c} S
$$

Where $F$ is the external force of PDMS, and $S$ is the relative cross section of PDMS. Combining Equation (4-6), we can deduce a conclusion that the main factor affecting the recovery deformation of SPCS is the relative cross-sectional area of PDMS, that is, the heterogeneous distribution of PDMS structure thickness.

In summary, through the above-mentioned mathematical theoretical analysis of the SPCS deformation mechanism, it can be known that the two key variables for SPCS to achieve periodic deformation are the temperature of SMA and the structure of PDMS. And the main factors affecting the realization of heterogeneity deformation is the heterogeneous distribution of PDMS structure thickness.

To further verify the feasibility of the SPCS deformation mechanism, we conducted the numerical analysis by using the software COMSOL Multiphysics 5.4. The simulation results of SPCS periodic and heterogeneous deformation was shown in Figure 2. As shown in the simulation Figure 2(a), the PDMS must have a certain thickness before the SPCS can complete the periodic deformation. On this basis, the different distribution forms of structures lead to the heterogeneity deformation of SPCS, as shown in Figure 2(b).

\subsection{Optimizing Periodic Actuation}

As shown in the "one-way deformation" of Figure 3(a), the one-way SMA wire will recover to the bending shape and cannot achieve the periodic deformation. With the advantages of integrated manufacturing, the flexible unit (PDMS) was introduced to prepare SPCS, and the PDMS store strain energy at high temperature. Then it released the strain energy at low temperature, deforming 
the SMA wire into a straight shape for the periodic deformation, as the " periodic deformation" of Figure 3(a) illustrated. Since the SPCS is thermally triggered to bend, and the strain potential energy forces a Periodic deformation, as shown in Figure 3(f). Therefore, the thermal triggered performance is an important index to evaluate the response characteristics. In this section, we first researched the heat Joule effect of SMA under various currents. As shown in Figure 3(b), it was noticed that increasing the current stimulus was able to promote the temperature elevation efficiency, especially the SPCS surface temperature reached as high as $54{ }^{\circ} \mathrm{C}$ in the case of $4 \mathrm{~A}$. Additionally, in the case the current was higher than $2.5 \mathrm{~A}$, SPCS surface temperature reached phase transformation temperature (i.e., $45^{\circ} \mathrm{C}$ ), even exceeded it. Given the effect that the heat transferred from SMA wire towards PDMS, we can reasonably assume that SMA wire temperature was higher than $45{ }^{\circ} \mathrm{C}$, which implied the phase transformation from Martensite phase into Austenite phase had finished. Then, we researched the SPCS periodic actuation under various current-induced heat Joule effect. The bending curves were recorded under various current ranging from $2 \mathrm{~A}$ to $4 \mathrm{~A}$, as shown in Figure 3(c). It was noticed that increasing the current promoted bending efficiency. In the case of 3.0 A, 3.5 A and 4.0 A, the response time for maximum bending angle relied on the temperature elevation efficiency. Under the higher current condition, the SPCS experienced a faster bending process and easier reaching maximum bending angle (i.e., $50^{\circ}$ ). However, the SPCS showed slowly increase in deformation under $2.0 \mathrm{~A}$ and $2.5 \mathrm{~A}$, the bending was far away from the maximum bending angle, which may be attributed to the effect that the SMA temperature was less than the phase transition completion temperature. Importantly, the SPCS could experience deformation recovery because of the strain potential energy release process. For the higher current condition, SPCS temperature could not dissipate due to the poor thermal conductivity of PDMS. The SPCS could not recover to its initial state due to the low heat 
dissipation attributed to the effect that SMA still at the phase transformation state, in which high stress compiled the deformation recovery process and resulted in a loss in the Periodic actuation, which was more obvious in the case of $4.0 \mathrm{~A}$ current. In the case of $2.0 \mathrm{~A}, 2.5 \mathrm{~A}$ and $3.0 \mathrm{~A}$ current, their final temperature enabled recovery to ambient temperature, which is lower than the Martensite transformation temperature $\mathrm{M}_{\mathrm{f}}$. In terms of the SMA phase diagram ${ }^{[45]}$, there is no stress existed inner SMA, which was helpful for the total deformation recovery during the strain potential energy release process.

Through the theoretical analysis of the SPCS deformation in section 3.2, it was concluded that PDMS thickness was the other important factor affecting the SPCS maximum bending angle. Increasing thickness of PDMS, although, played a positive role in enhancing the stiffness of the SPCS to hinder the mechanical bending of SPCS, but it enabled the deformation recovery more easily. Figure 3(d) recorded the SPCS bending process as the PDMS thickness varied from $2 \mathrm{~mm}$ to $6 \mathrm{~mm}$. It was noticed that these SPCS all experienced Periodic deformation, while differed in maximum bending angle and recovery deformation angle. Increasing the thickness of PDMS would induce bending angle decline and reached as high as $70^{\circ}$ in the case of $2 \mathrm{~mm}$ thickness while promoting the deformation total recovery. Especially, in the case the thickness was higher than $4 \mathrm{~mm}$, the SPCS could be remarkably restored to the initial state, which implied the Cauchy stresses stored inner PDMS was sufficiently to overcome the residual stress inside the SMA. For the decrease in the bending angle as the thickness of PDMS exceeded $4 \mathrm{~mm}$, it is the moment of inertia of the section played a decisive role in affecting the bending angle, as the inserted images shown in Figure 3(e). According to the classical material mechanical model, the moment of inertia of the section would experience an increased tendency with the augment of PDMS thickness, which could give rise to an attenuated bending angle under the same uniformly distributed load 
condition. Nevertheless, it could not be ignored that the mechanical bending demonstrated an insufficient recovery in the case of $2 \mathrm{~mm}$ and $3 \mathrm{~mm}$ PDMS thickness. It may be attributed to the effect that the internal stress generated by the mechanical bending exceeded the yield stress of PDMS, which caused partial plastic deformation inner the PDMS.

\subsection{Controllable and heterogeneous Deformation}

Combining Equation (4-6)in Section 3.2, there is a certain mapping relationship between the structural thickness distribution state of PDMS and the deformation properties of SPCS. Therefore, in this section, some forms of PDMS structure thickness distribution are selected arbitrarily to investigate their influence on SPCS deformation. First, as shown in Figure 4(a), in order to achieve deformation, we designed the "joints" as triangular groove structures with different angles and groove depths, and use $\alpha$ a to describe the "joints" deformation. The curves of the maximum deformation angle at the "joints" were shown in Figure 4(c). In the case of a $90^{\circ}$ triangular groove structure with a depth of $2 \mathrm{~mm}$, the "joint \#1" exhibited a $32^{\circ}$ deformation angle, while the deformation angle reached $36^{\circ}$ at "joint $\# 1$ " with a $120^{\circ}$ triangular groove structure. However, for a $90^{\circ}$ triangular groove structure with a depth of $4 \mathrm{~mm}$, the maximum deformation of "joint $\# 1$ " reached $35^{\circ}$, while the "joint \# 1 " with a $120^{\circ}$ triangular groove structure obtained a deformation angle of $38^{\circ}$. Therefore, as shown in Figure 4(b), both the groove angle and the groove depth had a positive effect on the deformation of "joints". In addition, the position of the groove structure is another important factor affecting the axial deformation of "joints". For a $90^{\circ}$ triangular groove structure with a depth of $4 \mathrm{~mm}$, the maximum deformation of "joint $\# 2$ " reached $32^{\circ}$, while the "joint \# 6" obtained a deformation angle of $23^{\circ}$. In the case of a $90^{\circ}$ triangular groove structure with a depth of $4 \mathrm{~mm}$, the maximum deformation of "joint $\# 2$ " reached $35^{\circ}$, while the "joint \# 6" obtained a deformation angle of $27^{\circ}$. Obviously, the amount of maximum deformation was also 
proportional to the distance from the root of the SPCS. The time curves required for joints to obtain the maximum deformation were given in Figure 4(d). For a $120^{\circ}$ triangular groove with $4 \mathrm{~mm}$ depth, "joint \# 1" only needs $1.2 \mathrm{~s}$ to obtain the maximum deformation, while "joint \# 6" requires 3.8s. However, for a $90^{\circ}$ triangular groove with $4 \mathrm{~mm}$ depth, it takes $5 \mathrm{~s}$ for "joint \# 6 " to obtain the maximum deformation. For a $3 \mathrm{~mm}$ groove depth, the time to obtain the maximum deformation of "joint \# 6" had also increased to 6s. Therefore, the time for "joints" to obtain the maximum deformation is also positively related to its distance from the root of the SPCS.

Based on the analysis of the above experimental results, we applied the mechanism of periodic heterogeneous deformation to the design of bionic fingers. It is well known that human fingers are composed of three phalanges interconnected with two knuckles. Due to the independent and cooperative bending of these two knuckles, two-stage curling can be complemented. In our proposed bionic finger, the two-stage deformation of SPCS was achieved by changing the angle of the triangular groove at joints. as shown in "\# 1 Two-stage deformation of SPCS" of Figure 4(a), the "finger joint" is designed as a flexible hinge to increase the possibility of deformation, and the rests are used as "phalangeal" to enhance the grip force of "soft finger". The middle joint was designed as a $120^{\circ}$ triangular groove, and the distal joint was a $90^{\circ}$ triangular groove. Applying an electric current to the SMA wire embedded within the actuator raises its temperature via Joule heating until its energization time reaches $15 \mathrm{~s}$ (Make sure the temperature exceeds the SMA austenite transition temperature). Figure 4(f) shows the whole deformation of "soft finger", it could be noticed that the maximum deformation at the "joints" was greater than that at the "phalanx". This is mainly due to the reduced stiffness of "joints", which makes the "joints" easier to deform (Movie S1). As shown in Figure 4(e), the maximum deformation at the "middle joint" reached $50^{\circ}$, which exceeding $36^{\circ}$ deformation at the "distal joint". It may be attributed to the 
difference in the gradient structure of the "middle joint" and "distal joint", which results in a difference in the stiffness distribution at the two joints. Moreover, the "middle joint" only needs $8 \mathrm{~s}$ to obtain the maximum deformation, but the "distal joint" takes $15 \mathrm{~s}$ to reach the maximum deformation. And, after the 80 s, both "distal joint" and "middle joint" can finally tend to recover to its initial state. However, the recovery speed of the "middle joint" is significantly higher than "distal joint" due to the larger strain potential energy stored in the "middle joint". Furthermore, the surface area on the "middle joint" is larger than that of the "distal joint", which bring higher temperature diffusion efficiency. In short, the internal temperature of the SMA in the intermediate joint can quickly drop below the phase transition state, which indicates that the internal stress of the SMA caused by Joule heat is almost eliminated, and no invalid loss of the strain potential energy. Through the above analysis, it was concluded that the structural optimization design of the strain potential energy storage component (PDMS) can not only play the role of the actuator twoway memory but also realize the heterogeneous deformation of the actuator. Meanwhile, the structural design of the strain potential energy storage element can also improve the local thermal diffusion efficiency of SMA, thereby enhancing the deformation efficiency.

\subsection{Soft gripper Demonstration}

The optimized design of the strain potential energy distribution state proposed for heterogeneous bending deformation has many potential applications in soft robotics. To show the functionality of this approach, inspired by the Human hands' concept, we designed and printed a soft gripper composed of "soft finger". Each "soft finger" followed the design of the periodic heterogeneous deformation actuators as outlined in Section 3.4. With the difference that each finger had two rows of embedded SMA wires with a $6 \mathrm{~mm}$ distance from each other. In addition, the five fingers are 
mounted in the metacarpal plate in a bionic hand pattern with each finger tightly arranged, the thumb sits in opposition side between the index finger and the ring finger.

The five fingers were connected in parallel and were individually actuated using an electrical current of $3 \mathrm{~A}$ regardless of the target object or grasp to control each finger independently, and the stiffness of each joint independently changed by the structural constraints. Therefore, we can control the independent movement of each joint through the way of current and structure interaction (Movie S2). The result of "soft hands" grasping objects of different shapes, such as cylinder (138g), cuboids (152g), and sphere-shaped objects (98g), are shown in Figure 5. As shown in Figure 5(a), for the cylinder-shaped objects, the "soft hand" can obtain a good grasping ability to grasp an object by adjusting the deformation angle of each joint adaptively. As grasping cuboid-shaped and sphere-shaped objects, the main considerations focused on the continuous heterogeneous deformation of "soft finger" under local structural constraints. Although the curvature was different, relatively continuous and adaptive shape-shifting could be accomplished. As shown in Figure 5(b) and (c), it can be seen that the gripper can grab objects with a large range of dimensions by adjusting the bending angles of both joints of each finger (Movie S3). The above experimental results demonstrate that the proposed structural SPCS can achieve Periodic bidirectional motion and heterogeneous, which will make it easily implemented as part of a robotic, even directly as a robotic application.

\section{Conclusions}

In summary, we presented a periodic heterogeneous actuated SMA-PDMS composite structure (SPCS) and described its structure, theoretical model, simulation, fabrication as well as the deformation performance test. Due to the electrothermal effect induced heat accumulation of SMA under the current stimuli, the shrinkage deformation can occur in SMA wire to actuate the SPCS 
bending deformation. Beneficial from the excellent elastic properties of PDMS, the Periodic deformation of the SPCS could be accomplished by the release of strain potential energy, and realize the heterogeneous deformation of SPCS through the structural constraints of PDMS. To analyze the whole deformation process of the SPCS, a theoretical model combing the heat transfer process inside SPCS, thermo-constitutive model of SMA and thermomechanical model of SPCS was constructed. The two core factors, current and PDMS structure, affecting the SPCS deformation have been proved through the theoretical model and simulation results based on the thermomechanical model of SPCS. We characterized the deformation performance of SPCS with various stimulation current. The increase of current improves the deformation efficiency of SPCS during the actuation process, while limits the deformation recovery speed due to the low heat dissipation rate under a higher temperature induced by the Joule heating effect. Besides, it can be concluded that the increase in the structural thickness of PDMS limits the mechanical deformation of the SPCS, but contributes to Periodic deformation, and the heterogeneous deformation of the SPCS can be achieved by partially structured PDMS, thereby increasing the complexity of SPCS deformation.

\section{Supporting Information}

Supporting Information is available from the Wiley Online Library or from the author.

\section{Acknowledgments}

This work is supported by National Natural Science Foundation of China (No. 91748209, 51705406), China Postdoctoral Innovation Talent Support Program (Grant No. BX20190272) and the Fund of National Engineering and Research Center for Commercial Aircraft Manufacturing (Grant No. COMAC-SFGS-2019-477). 


\section{Conflict of Interest}

The authors declare no conflict of interest

Keywords: Shape Memory Alloys, Soft Actuators, Strain potential energy distribution, Periodic Deformation, Heterogeneous Deformation

\section{References}

[1] Rus D, Tolley M T. Nature 2015, 521, 7553, 467-475.

[2] Jayaram K, Full R J. Proceedings of the National Academy of Sciences 2016, 113, 8, E950E957.

[3] Laschi C, Mazzolai B, Cianchetti M. Sci. Robot 2016, 1, 1, eaah3690.

[4] Wang W, Kim N G, Rodrigue H, et al. Materials Horizons 2017, 4, 3, 367-376.

[5] Gul J Z, Yang Y J, Su K Y, et al. Soft robotics 2017, 4, 3, 224-240.

[6] Rich S I, Wood R J, Majidi C. Nature Electronics 2018, 1, 2, 102-112.

[7] Martella D, Nocentini S, Parmeggiani C, et al. Advanced Materials Technologies 2019, 4, 2, 1800571.

[8] Wang W, Li C, Rodrigue H, et al. Advanced Functional Materials 2017, 27, 7, 1604214.

[9] Niu D, Jiang W, Ye G, et al. Smart Materials and Structures 2019, 28, 2, 02LT01.

[10] Shen Q, Trabia S, Stalbaum T, et al. Scientific reports 2016, 6, 24462.

[11] Li P, Wang Y, Gupta U, et al. Advanced Functional Materials 2019, 29,37, 1901908.

[12] Conn A T, Rossiter J, Smart Materials and Structures. 2012, 21 3, 035012

[13] Christianson C, Goldberg N N, Deheyn D D, et al. Science Robotics, 2018, 3, 17, eaat1893.

[14] Li T, Li G, Liang Y, et al. Science advances 2017, 3 4, E1602045

[15] Godaba H, Li J, Wang Y, et al. IEEE Robotics and Automation Letters 2016, 1, 2, 624-631.

[16] Mosadegh B, Polygerinos P, Keplinger C, et al. Advanced Functional Materials 2014, 24 15, 2163-2170.

[17] Shen H. Nature 2016, 530, 7588, 24. 
[18] Liu X, Duan Y, Hitzmann A, et al. Robotics and Autonomous Systems 2018, 110, 85-91.

[19] Marchese AD, Onal CD, Rus D. Soft robotics. 2014, 1 1,75-87

[20] Tan Y, Wang D, Xu H, et al. ACS applied materials \& interfaces, 2018, 10, 46, 40125-40131.

[21] Wei S, Lu W, Le X, et al. Angewandte Chemie International Edition 2019, 58,45, 1624316251.

[22] Xu T, Zhang J, Salehizadeh M, et al. Science Robotics 2019, 4, 29, eaav4494.

[23] Garstecki P, Tierno P, Weibel D B, et al. Journal of Physics: Condensed Matter 2009, 21, 20, 204110.

[24] Schmauch M M, Mishra S R, Evans B A, et al. ACS applied materials \& interfaces, 2017, 9, 13, 11895-11901.

[25] Li Z, Huang R, Liu Z. Advanced Materials Technologies 2019, 4,12, 1900653.

[26] Rodrigue H, Wang W, Han M W, et al. Soft robotics 2017, 4, 1, 3-15

[27] Ashir M, Nocke A, Theiss C, et al. Composite Structures. 2017, 170, 243-249.

[28] Huang X, Kumar K, Jawed M K, et al. Science Robotics 2018, 3,25, 7557.

[29] Jin H, Dong E, Xu M, et al. Smart Materials and Structures 2016, 25 8, 085026

[30] Kim H I, Han M W, Song S H, et al. Composites Part B: Engineering. 2016, 105: 138-148.

[31] Huang X, Kumar K, Jawed M K, et al. Advanced Materials Technologies 2019, 4 4, 1800540.

[32] Coral W, Rossi C, Curet O M, et al. Bioinspiration \& biomimetics 2018, 13, 5, 056009.

[33] Jin H, Dong E, Alici G, et al. Bioinspiration \& biomimetics, 2016, 11, 5, 056012.

[34] Kim H S, Lee J Y, Chu W S, et al. Soft robotics 2017, 4, 1, 49-60.

[35] Zhang S, Liu B, Wang L, et al. IEEE/ASME transactions on mechatronics 2014, 19, 6, 17731785.

[36] Akbari S, Sakhaei A H, Panjwani S, et al. Sensors and Actuators A: Physical 2019, 290, 177 189.

[37] Rodrigue H, Wei W, Bhandari B, et al. Smart Materials and Structures 2015, 24, 12,125003.

[38] Rodrigue H, Wang W, Bhandari B, et al. Composites Part B: Engineering 2015, 82, 152-158.

[39] Wang W, Rodrigue H, Kim H I, et al. Composites Part B: Engineering 2016, 98, 397-405.

[40] Kök M, Dağdelen F, Aydoğdu A, et al. Journal of Physics: Conference Series. IOP Publishing 2016, 667, 1, 012011.

[41] Tanaka K. Journal of Pressure Vessel Technology 1990,112 2,158-163 
[42] Yu C, Kang G, Kan Q, et al. International Journal of Plasticity 2015, 72, 60-90.

[43] Sakhaei A H, Thamburaja P. Mechanics of Materials 2017, 109, 114-134.

[44] Ogden R W. Proceedings of the Royal Society of London. A. Mathematical and Physical Sciences 1972, 326, 1567, 565-584.

[45] Cortez-Vega R, Chairez I, Luviano-Juárez A, et al. Measurement 2018, 114, 340-353.

(a)

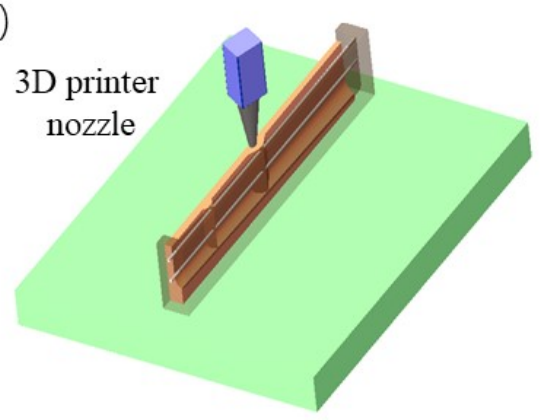

Integrated manufacturing of PDMS structure molds

(d)

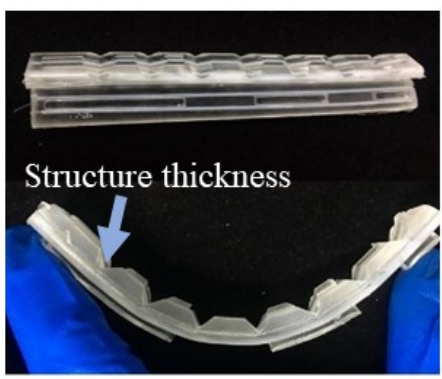

Heterogeneous distribution of PDMS structure (b)

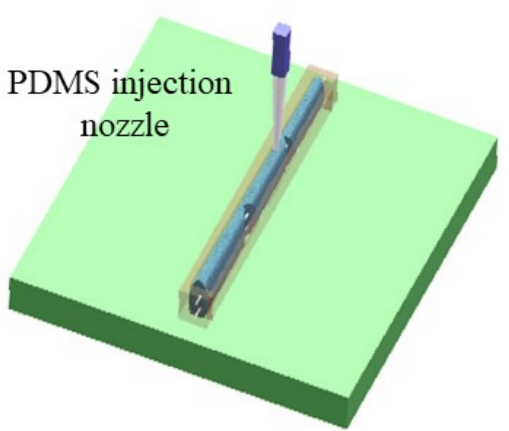

Continuous injection molding of PDMS structures

(e)

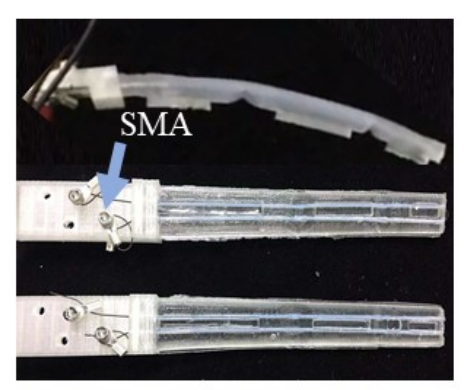

SPCS assembled by SMA and PDMS (c)

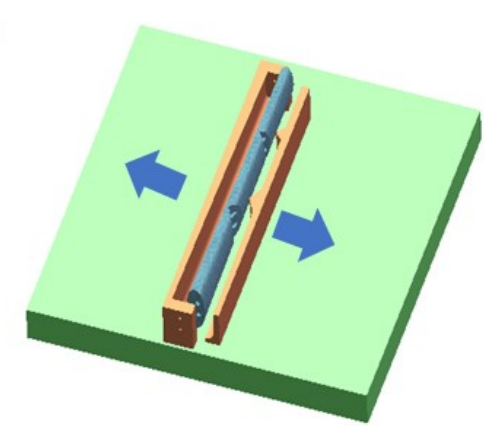

Take out the PDMS with structures

(f)

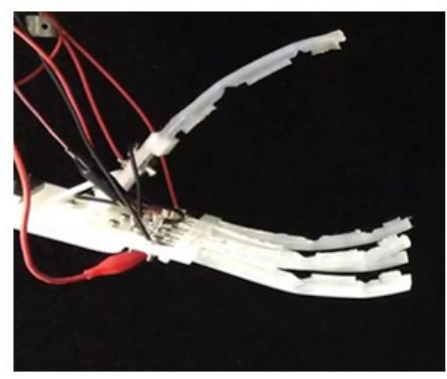

Soft gripper assembled by SPCS

Figure 1 A multi-stage fabrication process of SPCS based on integrating 3D printing technology and automatic dispensing method. 


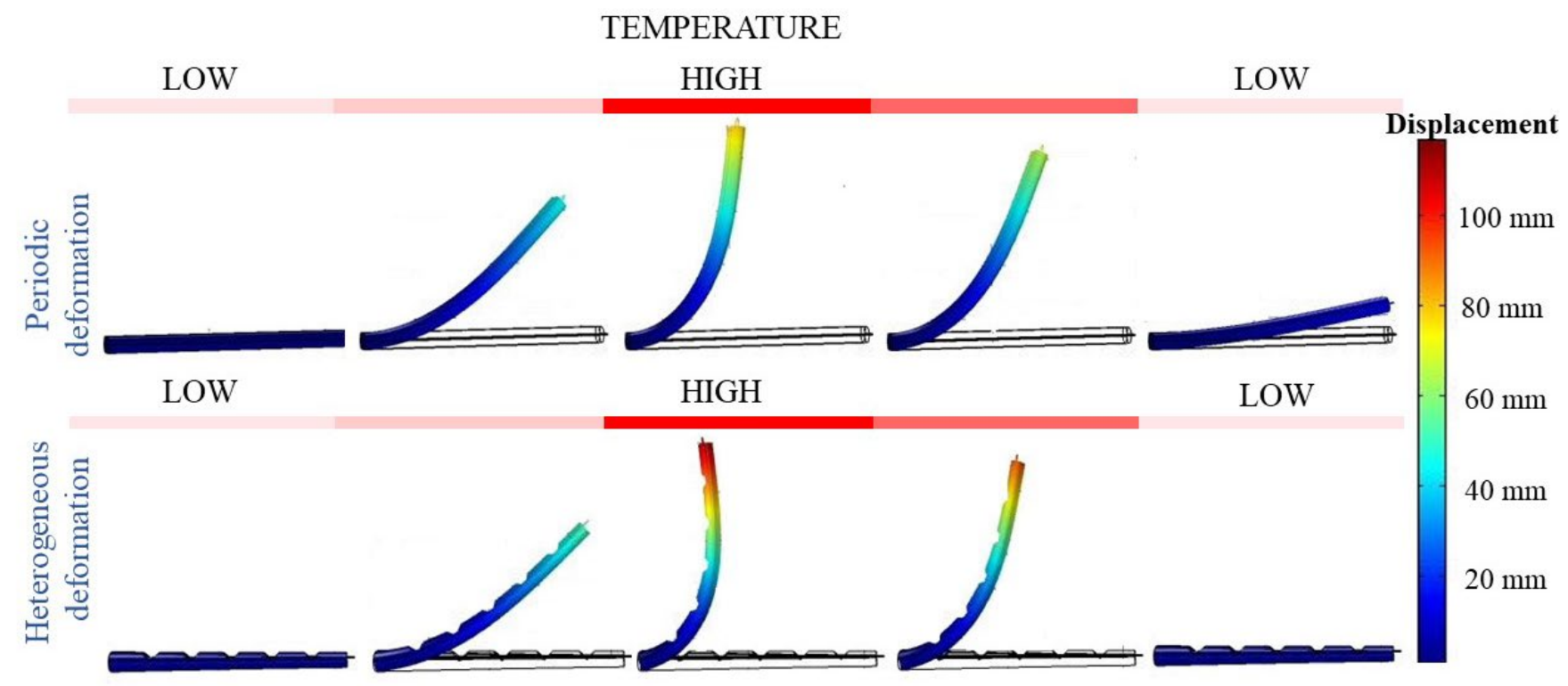

Figure 2 The simulation diagram of the SPCS deformation process. (a). Periodic deformation process of SPCS. (b). The multistage heterogeneous deformation process of SPCS. 
(a)

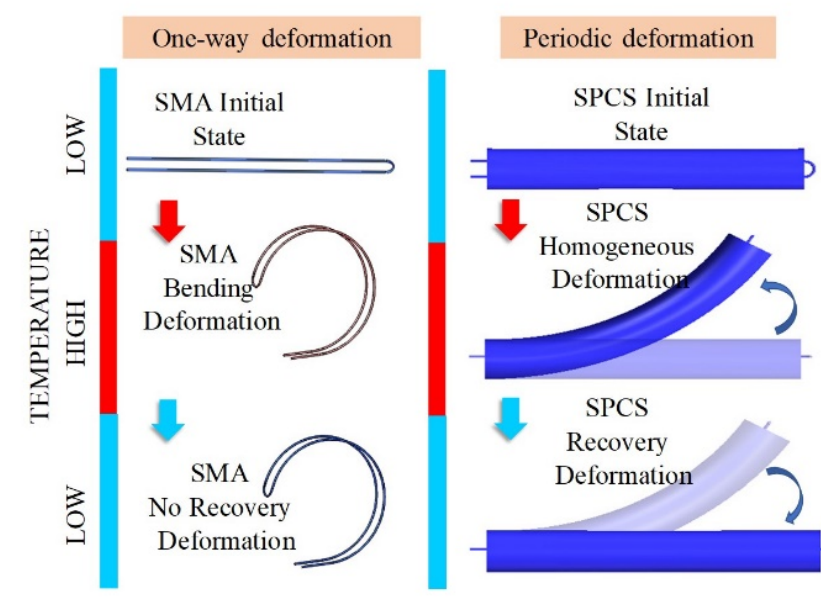

(c)

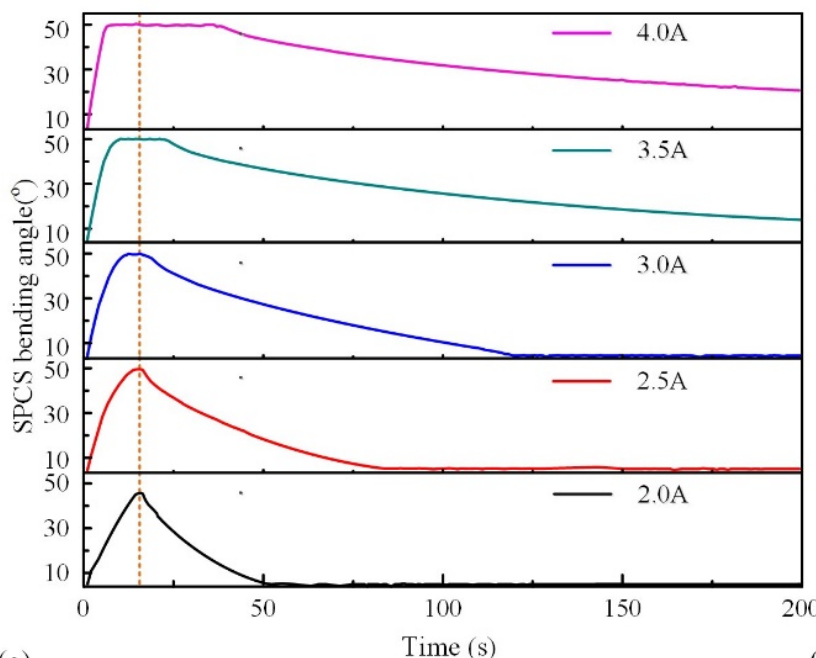

(e)

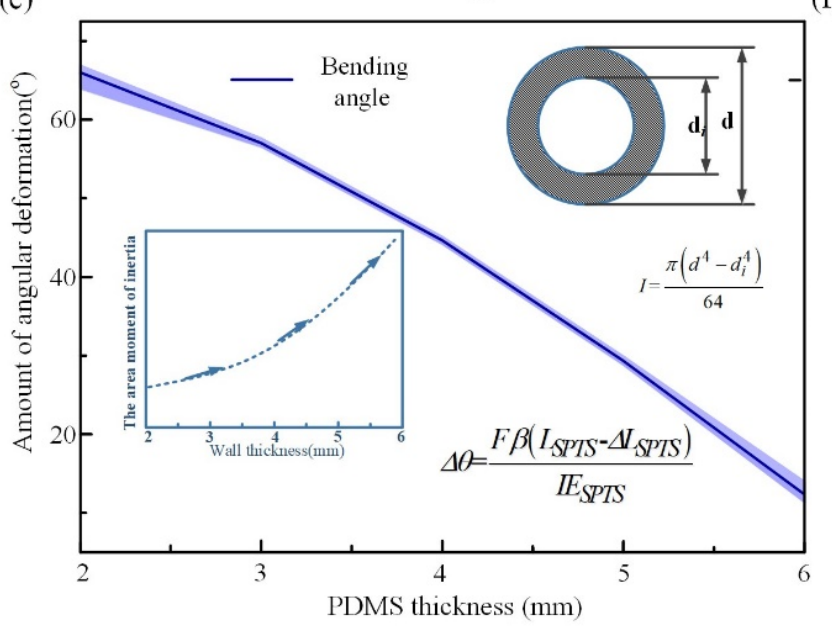

(b)
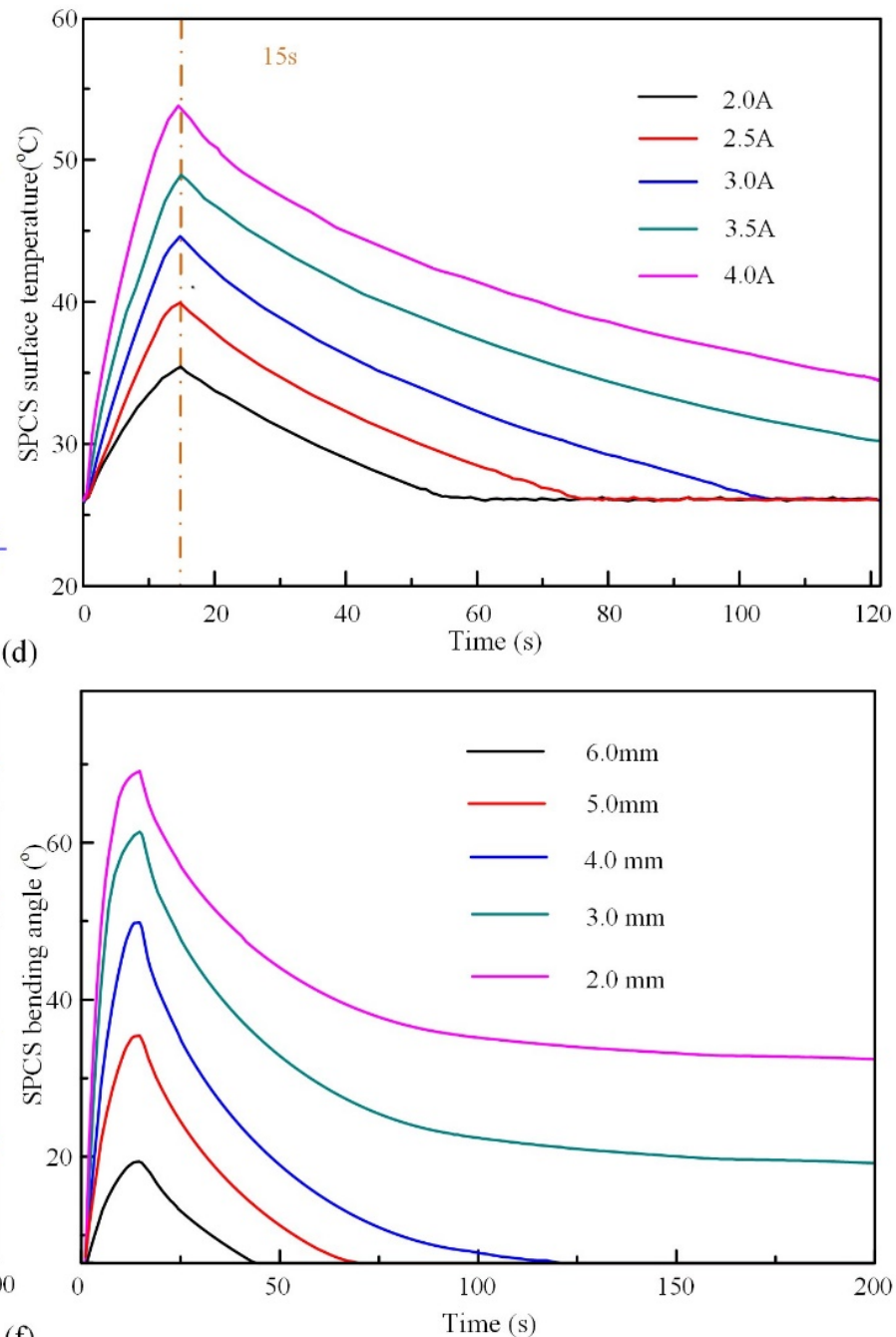

(f)

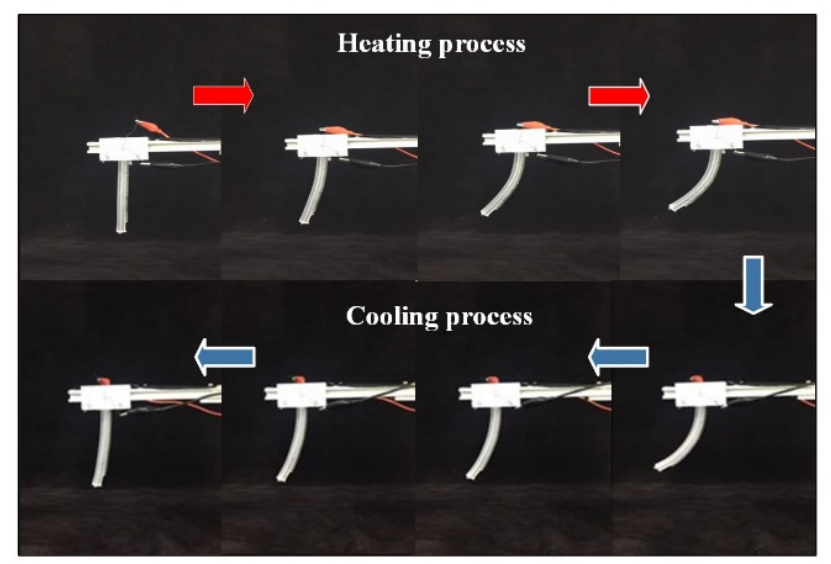


Figure 3 (a) Mechanism introduction. (b) Temperature profiles of SPCS at an energized current of 2.0 A to 4.0 A.(c) Bending angle as a function of time for various currents. (d) Bending angle as a function of time for various wall thicknesses. (e) The effect of PDMS thickness on SPCS maximum bending angle. (f) The bending deformation process of SPCS. 
(a)
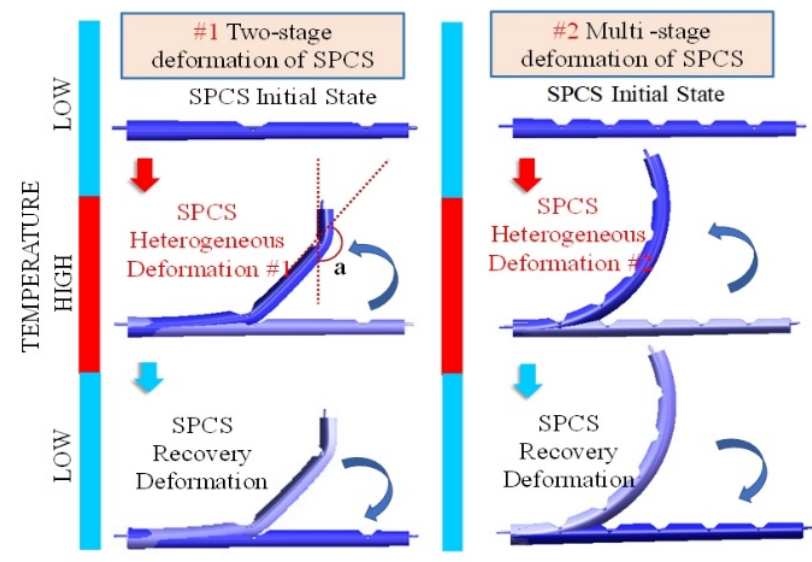

(c)
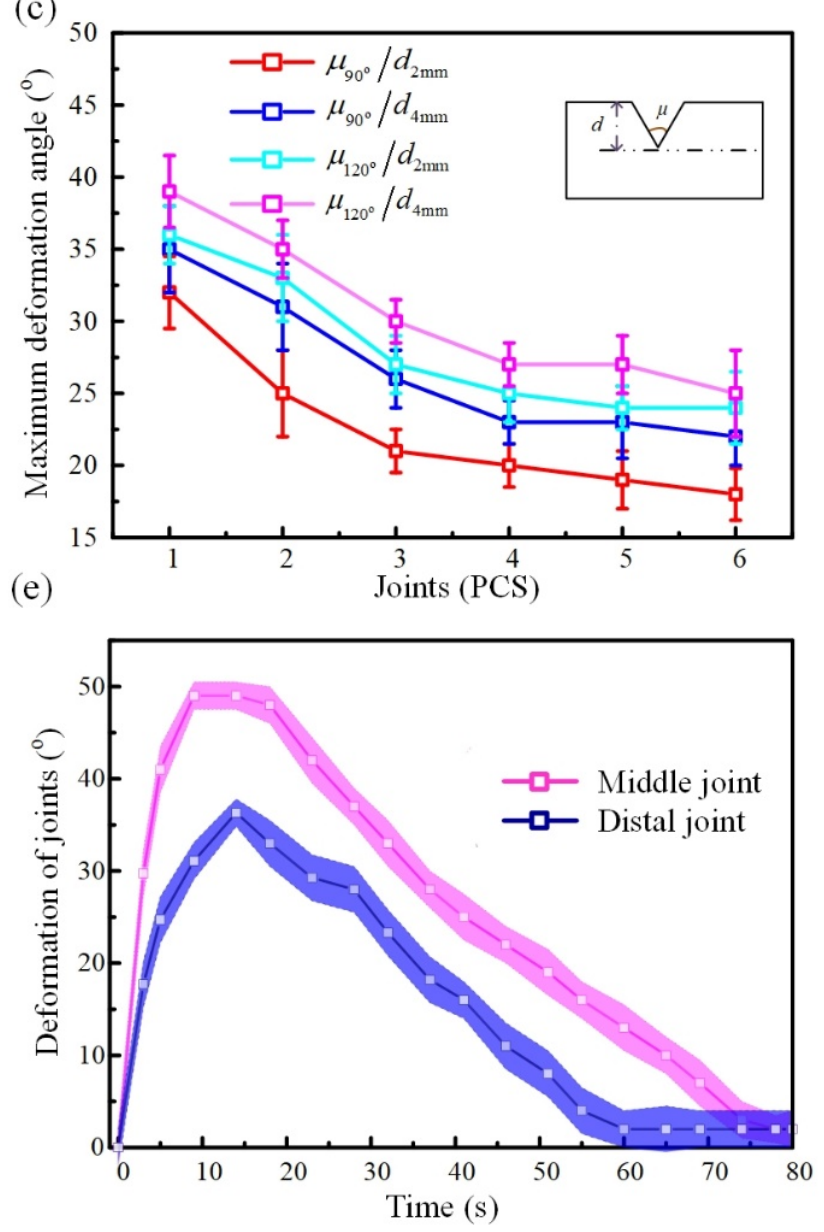

(b)

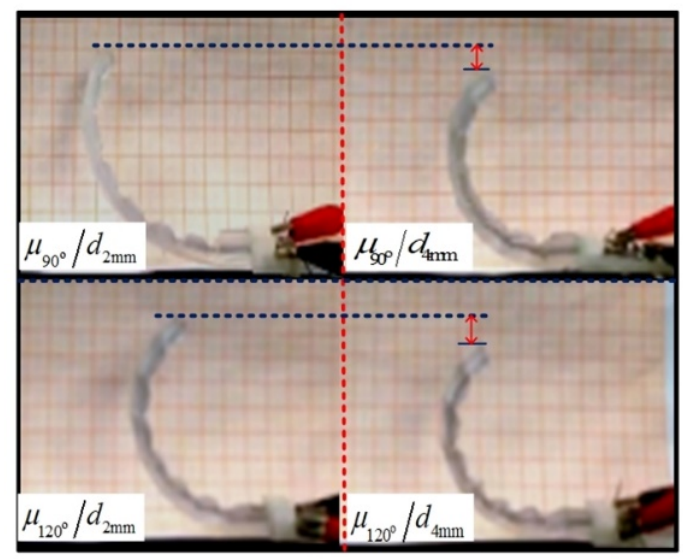

(d)

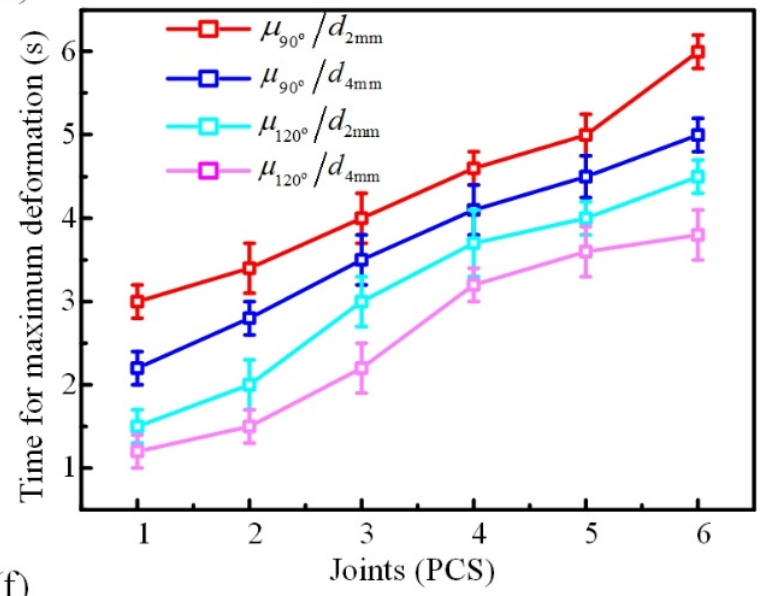

(f)

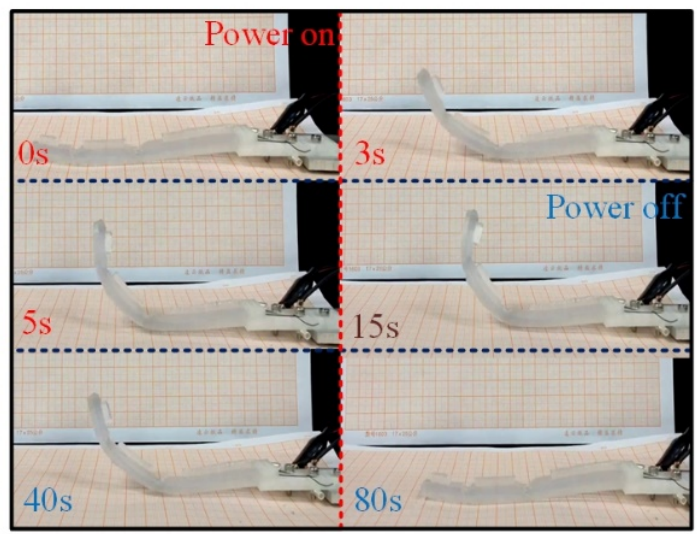

Figure 4 (a) Mechanism introduction. (b)The maximum deformation of SPCS with structured "multi-joints". (c) The curves of the relationship between the maximum deformation angle and “joint". (d) The time required for "multi-joints" to achieve maximum deformation various "joints". 
(e) The deformation as a function of time for two joints. (f) Multi-deformation process of "soft finger".

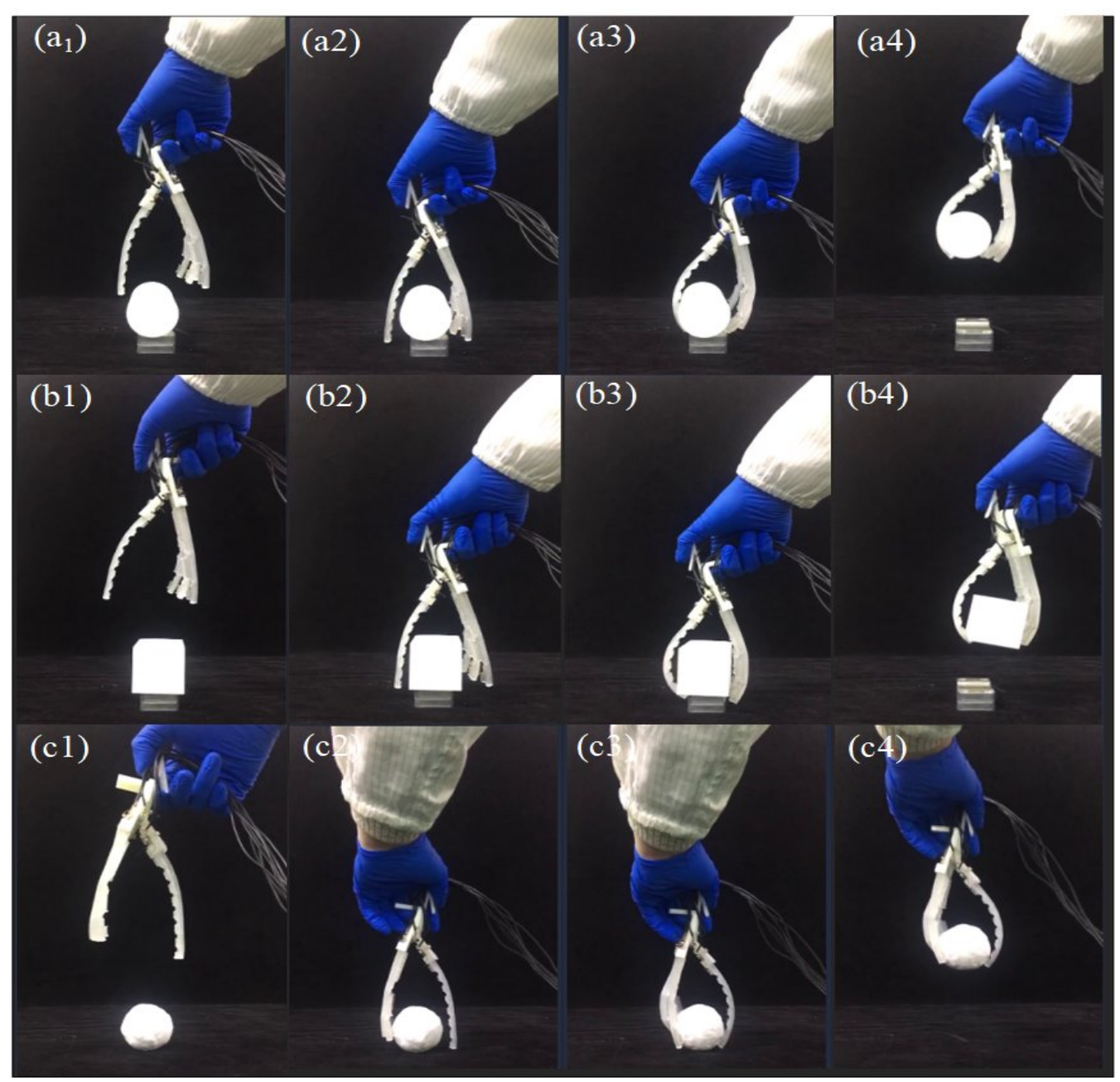

Figure 5 Photographs showing the grasping of various objects by the soft robotic hand. $\left(a_{1}\right)-\left(a_{4}\right)$ cylinder-shaped (138g), ( $\left.b_{1}\right)-\left(b_{4}\right)$ cuboid-shaped shaped object $(152 \mathrm{~g}),\left(c_{1}\right)-\left(c_{4}\right)$ sphere-shaped object $(98 \mathrm{~g})$. 